\title{
Development of a vanillate biosensor for the vanillin biosynthesis pathway in $E$. coli
}

\author{
Aditya M. Kunjapur*1,2, Kristala L. J. Prather*1
}

1. Department of Chemical Engineering, Massachusetts Institute of Technology, 77 Massachusetts

Avenue, Cambridge, MA 02139

2. Present Address: Department of Chemical and Biomolecular Engineering, University of Delaware, 150

Academy Street, Newark, DE 19716

* Correspondence to: Aditya M. Kunjapur (kunjapur@udel.edu) or Kristala L. J. Prather (lijp@mit.edu)

\begin{abstract}
Genetically encoded small molecule sensors can facilitate metabolic engineering by enabling highthroughput detection of metabolite concentrations, directed evolution of host and pathway enzymes, and dynamic regulation. The engineered de novo vanillin biosynthesis pathway assembled in Escherichia coli is industrially relevant and ideal for biosensor deployment given that the pathway requires only three heterologous enzyme-catalyzed reactions, generates naturally occurring metabolites, and may benefit from dynamic regulation. However, pathway flux is stalled and diverted by the activity of the Homo sapiens catechol $\mathrm{O}$-methyltransferase, which is intended to catalyze the conversion of protocatechuate to vanillate. To confront this challenge, we constructed and applied a vanillate sensor based on the Caulobacter crescentus VanR-VanO system. Using components from a previously characterized E. coli promoter library, we achieved greater than 14 -fold dynamic range in our best rationally constructed sensor. We characterized sensor substrate specificity and found that this construct and an evolved variant are remarkably selective, exhibiting no detectable response to the regioisomer byproduct isovanillate. We then harnessed the evolved biosensor to conduct rapid bioprospecting of natural catechol $O$ -
\end{abstract}


methyltransferases. We identified eight that appear to have greater desired activity than the originally used variant, including three previously uncharacterized $O$-methyltransferases. Collectively, these efforts enrich our knowledge of how biosensing can aid metabolic engineering and constitute the foundation for future improvements in vanillin pathway productivity.

\section{Keywords}

Biosensor, Metabolic engineering, Vanillin, Vanillate, $O$-methyltransferase, Bioprospecting 


\section{Introduction}

Nature evolved a myriad of genetically encoded small molecule biosensors for detection and response to chemical stimuli. Allosteric transcription factors are a common form of biosensor and are commonly employed in inducible gene expression systems ${ }^{1}$ and in engineered genetic circuits ${ }^{2}$. The potential value of biosensors to metabolic engineers, who seek to maximize production of value-added products by manipulating cellular metabolism, is immense and multifaceted ${ }^{3,4}$. Genetically encoded biosensors facilitate metabolic engineering by enabling high-throughput detection of metabolite profiles, directed evolution of host and pathway enzymes ${ }^{5-7}$, and dynamic regulation ${ }^{8-10}$.

The $E$. coli de novo vanillin biosynthesis pathway ${ }^{11,12}$ is a highly industrially relevant pathway for which genetically encoded biosensors could be present for all three heterologous metabolites, and its potential as a testbed for multiplexed biosensing increases its academic relevance. Vanillin is the primary molecule responsible for vanilla flavor and is the largest flavor additive by volume, with an annual global market worth $\$ 650$ million and a total volume of 18,000 tons $^{13}$. It is also used as an intermediate for pharmaceuticals and specialty chemicals ${ }^{14}$. Notably, the vanillin biosynthesis pathway consists of three heterologous enzymes but numerous potential byproducts are also possible, which can be difficult to separate (Fig. 1). The enzyme AsbF from Bacillus thuringiensis catalyzes the conversion of an endogenous intermediate of the aromatic amino acid biosynthesis pathway, 3-dehydroshikimate, to protocatechuate (also referred to as 3,4-dihydroxybenzoate) ${ }^{15}$. The soluble catechol O-methyl-transferase from Homo sapiens $\left(\mathrm{OMT}_{H s}\right)$ catalyzes the next desired conversion of protocatechuate to vanillate ${ }^{16-18}$. Finally, the carboxylic acid reductase from Nocardia iowensis $\left(\operatorname{Car}_{N i}\right)$ catalyzes the final desired conversion of vanillate to vanillin ${ }^{19,20}$. Use of an engineered $E$. coli host (the RARE strain) minimizes rapid endogenous conversion of vanillin to vanillyl alcohol ${ }^{11}$. However, other products are commonly formed downstream of the protocatechuate branch point due to the broad substrate specificity of $\mathrm{OMT}_{H s}$ and $\mathrm{Car}_{N i}$. $\mathrm{OMT}_{H S}$ catalyzes the formation of isovanillate by transferring a methyl group to the hydroxy group at the para rather than 
meta position. $\operatorname{Car}_{N i}$ can also convert protocatechuate to protocatechualdehyde, which is a toxic intermediate, though it is possible for $\mathrm{OMT}_{H s}$ to subsequently convert protocatechualdehyde into vanillin.

The ability to sense vanillate could improve vanillin titers through several means. Based on the observation that protocatechuate accumulates, we previously found that $\mathrm{OMT}_{H s}$ steadily loses activity during the first 48 hours after induction and that the co-factor S-adenosyl-methionine (SAM), which is the required methyl donor, appears to become scarce as fermentation time proceeds ${ }^{12}$. A vanillate sensor could be used to improve $\mathrm{OMT}_{H s}$ activity, to engineer a host for increased SAM pool size, or to rapidly screen the natural diversity of OMTs in search of a better variant to relieve this pathway bottleneck. In principle, a vanillate sensor could also be used to delay expression of Car $_{N i}$ until vanillate accumulated, which should favor flux through desired heterologous reaction steps and decrease formation of toxic protocatechualdehyde.

We examined the literature for allosteric transcription factors that respond to the three desired heterologous metabolites - protocatechuate, vanillate, and vanillin - given that they are naturally obtained through lignin degradation (Fig. 1B). An E. coli sensor for protocatechuate was previously constructed by importing the PcaU activator and associated promoter sequence from Acinetobacter ${ }^{21}$. This sensor was reported to exhibit a graded (or analog) response across millimolar concentrations of protocatechuate, with high substrate specificity and approximately 10 -fold dynamic range. In addition to the protocatechuate sensor, multiple efforts have been undertaken to develop an E. coli sensor specific for vanillin. The QacR transcriptional repressor was computationally redesigned to bind to the non-native substrate vanillin and led to variants that exhibited dose-dependent de-repression in vitro and in vivo ${ }^{22}$. Additionally, recent work has revealed that a native E. coli promoter may be responsive specifically to vanillin ${ }^{23}$. However, we found no dedicated effort to construct and characterize a sensor responsive to vanillate in E. coli. 
Here, we report the development, characterization, and application of a genetically encoded sensor for vanillate in E. coli based on the Caulobacter crescentus VanR-VanO system. Our initial results obtained through rational modifications demonstrate the respective influence of repressor promoter strength and operator site positioning on sensor dynamic range. Our subsequent results showcase the exquisite molecular specificity of the VanR transcription factor through the dose-responses obtained for original and evolved vanillate biosensor constructs. We conclude by illustrating the value of biosensorbased bioprospecting by screening the natural diversity of catechol $O$-methyltransferases sampled across all domains of life. Our approach rapidly identifies variants that appear to have higher desired activity than $\mathrm{OMT}_{H s}$, including three previously uncharacterized $O$-methyltransferases.

\section{Results and Discussion}

Vanillate-responsive transcriptional repressors (VanR) exist in several bacteria, including Acinetobacter $^{24}$, Caulobacter ${ }^{25}$, Corynebacterium ${ }^{26}$, Myxococcus $^{27}$, and Pseudomonas ${ }^{28}$. These VanR variants bind to different operator (VanO) sites consisting of inverted repeats ranging from AACTAACTAA $\left(\mathrm{N}_{4}\right)$ TTAGGTATTT in Cornyebacterium glutamicum to ATTGGATCCAAT in Caulobacter crescentus (Fig. 2A/B). We sought to import the $C$. crescentus system into $E$. coli given the relatively small operator site and because a vanillate-inducible expression system was successfully developed for use in C. crescentus $^{25}$. At first, we considered the three base pair overlap of the natural VanO sequence with the C. crescentus -10 site a potential obstacle to direct promoter transfer and sought to test rational alternatives for E. coli (Fig. 2C). Placement of the VanO site at the same position in relation to the E. coli 10 site (leading to TATATT instead of the consensus TATAAT ${ }^{29}$ ) is predicted to significantly disfavor interaction with the E. coli sigma factor $70^{30}$.

We aimed to construct a functional vanillate sensor in E. coli using the C. crescentus VanR-VanO system to regulate expression of a red fluorescent protein (mRFP) (Fig. 2D). The complete list of candidate 
promoter sequences used for expression of codon-optimized VanR and mRFP are shown in Fig. 2E. Our initial vanillate sensor construct (pAMK-vansens-0) used the native $C$. crescentus promoter upstream of VanR to drive its expression. A strong Anderson promoter flanked by VanO sequences was placed upstream of mRFP. E. coli harboring this construct showed little to no change in mRFP fluorescence upon addition of $1 \mathrm{mM}$ vanillate. As we began to increase expression of VanR using Anderson library promoters instead of the native $C$. crescentus promoter (previously valued at $0.51 / 0.70 / 0.86$ expression strength pAMK-vansens-1/2/3, respectively), we observed an increased ratio of fluorescence in the presence of vanillate to fluorescence in the absence in the absence of vanillate (Fig. 3A). However, we observed substantial leaky fluorescence (Fig. 3A). After realizing that the downstream VanO site was neither adjacent to nor overlapping with the -10 site, we generated three additional constructs with the VanO site placed adjacent (pAMK-vansens-4) or with 1 or 2 bp overlap (pAMK-vansens-5 or pAMK-vansens-6, respectively). E. coli harboring these constructs exhibited low fluorescence in the absence of vanillate and increasing dynamic range as overlap of VanO and -10 site increased (Fig. 3B). The best performing construct, pAMK-vansens-6, exhibited a dynamic range of approximately 14 -fold and was chosen for further characterization.

We characterized the dose-response behavior of this vanillate sensor for five relevant substrates that are typically produced after co-expression of vanillin pathway genes in E. coli: protocatechuate, vanillate, isovanillate, protocatechualdehyde, and vanillin. These substrates were provided at concentrations ranging from $0.01 \mathrm{mM}$ to $1 \mathrm{mM}$, the latter of which is in the order of magnitude of observed accumulation of protocatechuate and vanillate in the absence of Car $_{N i}$ expression ${ }^{31}$. Furthermore, we compared vanillate sensor performance with the best available sensor reported for protocatechuate as a reference and to explore future potential for dual sensing. We cloned the published protocatechuate sensor (PcaU and promoter region) upstream of mRFP in place of equivalent components in the vanillate sensor to create an otherwise identical construct (pAMK-protosens-1). Based on an 
endpoint assay 24 hours after substrate supplementation, we observed graded dose-response to protocatechuate consistent with the literature ${ }^{21}$ (Fig. 4A). Unfortunately, the protocatechuate sensor also activated in the presence of vanillate, which had been reported. In contrast, our best vanillate sensor (pAMK-vansens-6) exhibited high sensitivity to vanillate, with half-maximal activation occurring below 10 $\mu \mathrm{M}$ and full activation below $100 \mu \mathrm{M}$ range (Fig. 4B). The high sensitivity and more quickly saturating response were concerning given our aspirations to ascertain relative concentration resulting from biosynthesis in different strains rather than merely whether vanillate was present. Our concerns were mitigated by the excellent specificity of the vanillate sensor, which exhibited minimal response to protocatechuate and remarkably no response to the regioisomer isovanillate. Many catechol $O$ methyltransferases, including $\mathrm{OMT}_{H s}$, possess the undesired capacity to methylate a hydroxy group on the para rather than meta position of the aromatic ring relative to the carboxylic acid group, which gives rise to isovanillate from protocatechuate ${ }^{17,32}$. Isovanillate is particularly problematic given that Car $_{N i}$ will convert it to isovanillin, and these two regioisomers add hurdles to separation from vanillate and vanillin for accurate quantification or product purification.

We next tested the vanillate sensor in cells that expressed the vanillin biosynthesis pathway. $E$. coli RARE cells harboring pathway constructs and pAMK-vansens- 6 exhibited a low level of fluorescence in LB medium and several fold additional activation in response to externally supplied vanillate (SI Fig. 1A). These cells also demonstrated endogenous activation of the vanillate sensor in M9 minimal media supplemented with glucose and IPTG for induction of pathway genes (SI Fig. 1B). However, the sensor exhibited full activation under this condition and full activation when no IPTG was added, suggesting that the sensor is saturated by leaky uninduced pathway expression in the presence of glucose.

To overcome these challenges, we pursued further engineering of our vanillate sensor. We provided colleagues in the laboratory of Professor Christopher Voigt (MIT Department of Biological Engineering, Cambridge, MA) this sensor as well as the recloned protocatechuate sensor for evolution by 
compartmentalized partnered replication ${ }^{33}$, which was successfully used to improve the dynamic range of these two sensors as well as ten others as very recently reported ${ }^{34}$. As before, we investigated the doseresponse and substrate specificity of these evolved sensors, which drive expression of YFP instead of mRFP

(Fig. 4C and 4D). We observed improvements in dynamic range and protocatechuate sensor specificity consistent with the literature ${ }^{34}$. Fortunately, the evolved vanillate sensor, which includes mutations to VanR, maintains an absence of response to isovanillate. Although the evolved vanillate sensor was originally found to have increased cooperativity relative to the original vanillate sensor, we found over repeatable experiments in our hands that the dose-response of the evolved vanillate sensor was more graded than the original vanillate sensor and that it saturated at a higher vanillate concentration. Both features increase biosensing efficacy for metabolic engineering contexts. Th discrepancy may be a function of media conditions, supplementation time, and/or assay endpoint time.

Given that the evolved vanillate sensor can generate proportional fluorescence at sub-millimolar vanillate concentrations, has extremely low background fluorescence, and does not respond to related vanillin pathway molecules including isovanillate, we harnessed it to improve the vanillin pathway by biosensor-based bioprospecting. As discussed earlier, we previously identified $\mathrm{OMT}_{H s}$ as the bottleneck for pathway flux and hypothesized that other $O$-methyltransferases may be better at catalyzing vanillate formation, especially in a bacterial host. As a starting point for sampling the natural diversity of $O$ methyltransferases, we considered all relevant literature including published patent applications. One patent application filed by Evolva S.A. and International Flavors and Fragrances, Inc. (IFF) describes the testing of different $O$-methyltransferases expressed in yeast and displays data for roughly 20 natural variants $^{32}$. We compiled a shortlist consisting of the best $O$-methyltransferases reported and then used protein BLAST on the $\mathrm{NCBI}$ nucleotide collection to identify putative enzymes and other $O$ methyltransferases that had not been previously tested. We sampled across the tree of life but biased our 
final candidate list of sixteen towards bacterial, fungal, and archaeal sources given our bacterial host (Fig. 5).

We screened these $O$-methyltransferases by co-transforming constructs harboring the evolved vanillate sensor and constructs harboring each of the library members into the E. coli RARE $\Delta m e t J \operatorname{strain}^{12}$. The deletion of the MetJ regulator of methionine biosynthesis is one of several modifications previously shown to improve conversion of protocatechuate to vanillate, presumably due to increased SAM pool size. We tested these cultures with IPTG supplementation at inoculation, which induces $O$ methyltransferase expression, and with or without $4 \mathrm{mM}$ protocatechuate supplementation. We then measured final OD and normalized fluorescence (YFP/OD) 24 hours after inoculation. Our first observation was that one of the variants grew substantially more slowly than the rest and achieved a lower final OD by approximately two- to three-fold (Fig. 6A). This variant, the $O$-methyltransferase from Phytophthora infestans or $\mathrm{OMT}_{P i}$, also exhibited the highest normalized fluorescence despite its potential toxicity. Because the expression of $\mathrm{OMT}_{p_{i}}$ resulted in a lower final OD independent of exogenous protocatechuate provision, the toxicity of $\mathrm{OMT}_{p i}$ expression may be unrelated to its high enzymatic activity or may be due to activity on other endogenous substrates. Given its adverse effect in vivo, this enzyme may be better suited to in vitro strategies for vanillin biosynthesis ${ }^{35}$ if high protein yields can be obtained.

The results of our screen suggest that eight $O$-methyltransferases could have greater desired in

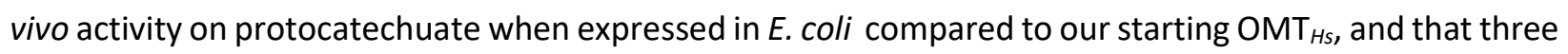
previously uncharacterized O-methyltransferases may have utility for this pathway and for broader biotechnology applications. Of the six best performing OMTs screened, the variants from Phytophthora infestans (top performing) and Candida orthopsilosis (third best performing) are described in the patent application filed by Evolva and IFF ${ }^{32}$. The second best performing OMT is from Synechocystis sp. PCC 6714 and is previously uncharacterized to the best of our knowledge. A report describing the draft genome sequence of PCC 6714 identified a genomic island containing a putative methyltransferase upon 
comparative genome analysis of the related Synechocystis sp. PCC 6803 strain $^{36}$. The complete genome sequence record for PCC 6714 contains gene annotation of "Predicted O-methyltransferase YrrM" based on function prediction from protein sequence similarity ${ }^{37}$. Our finding that the PCC 6714 variant is so active is particularly interesting given the lack of activity observed from the PCC 6803 variant, which shares $86 \%$ protein sequence identity. When previously expressed in E. coli, the PCC 6803 variant exhibited preference for trihydroxylated flavones and reduced affinity for protocatechuate $\left(\mathrm{k}_{\mathrm{cat}} / \mathrm{K}_{\mathrm{M}}=37 \mathrm{M}^{-1} \mathrm{~s}^{-1}\right)^{38}$. Myxococcus xanthus is the source of our fourth best performing OMT, which is known to prefer protocatechuate as a substrate $\left(\mathrm{k}_{\mathrm{cat}} / \mathrm{K}_{\mathrm{M}}=5.5 \times 10^{3} \mathrm{M}^{-1} \mathrm{~s}^{-1}\right)^{39}$ and to have high regioselectivity for the meta position of protocatechuate despite having high regioselectivity for the para position of other substrates ${ }^{40,41}$. Like the OMT from PCC 6714, the fifth and sixth best performing OMTs, which are from uncultured marine thaumarchaeote KM3_66_E12 ${ }^{42}$ and from Myxosarcina sp. GI1 respectively, have not been characterized and are annotated as "Predicted O-methyltransferase YrrM".

Overall, this work seeks to overcome a critical limitation of the industrially relevant vanillin biosynthesis pathway through the development, characterization, and application of a genetically encoded biosensor for the heterologous intermediate vanillate. Biosensing in E. coli was successfully achieved by importing transcriptional regulator and operator components from $C$. crescentus and by introducing rational modifications in promoter sequences. The vanillate biosensor was then further evolved by directed evolution, and these biosensors along with similar biosensors for protocatechuate were evaluated for their substrate specificity and dose-response. Three of these sensors - the original vanillate sensor and the evolved vanillate and protocatechuate sensors - each exhibited sufficiently high specificity to enable their future use for improving the vanillin pathway. We demonstrated the value of the vanillate biosensor by using it to rapidly bioprospect alternative $O$-methyltransferases and our screen suggests that several variants will outperform our original choice, including three previously uncharacterized proteins. The effort described here lays the foundation for subsequent vanillin pathway 
bioRxiv preprint doi: https://doi.org/10.1101/375287; this version posted July 23,2018 . The copyright holder for this preprint (which was not certified by peer review) is the author/funder. All rights reserved. No reuse allowed without permission.

improvements as well as the ability to investigate the value of multiplexed biosensing of all desired heterologous metabolites in the vanillin pathway. 


\section{Methods}

Strains and Plasmids

E. coli strains and plasmids used in this study are listed in SI Table 1. Molecular biology techniques were performed according to standard practices $^{43}$ unless otherwise stated. Molecular cloning, vector propagation, and the majority of biosensor characterization were performed in $\mathrm{DH} 5 \alpha$. The host strain used for vanillin biosynthetic pathway co-expression experiments was the E. coli RARE strain (Addgene Catalog \#61440) ${ }^{11}$, which is derived from E. coli K-12 MG1655(DE3). Oligonucleotides were purchased from Sigma and are listed in SI Table 2. Q5 High Fidelity DNA Polymerase (New England Biolabs, MA) was used for DNA amplification.

The VanR and PcaU genes were synthesized as codon-optimized gBlocks (Integrated DNA Technologies, CA) and their sequences are included in SI Table 3. Original sensor constructs were cloned into the Duet vector system (Novagen, WI) using restriction digest-based cloning. Restriction enzymes and T4 DNA ligase were purchased from New England Biolabs. Propagated constructs were purified using a QIAprep Miniprep Kit (Qiagen, CA) and agarose gel fragments were purified using a Zymoclean Gel DNA Recovery Kit (Zymo Research, CA). All constructs were confirmed to be correct by nucleotide sequencing (Genewiz, NJ).

Constructs for biosensor-based screening of natural $O$-methyltransferase diversity were generated using Gibson assembly ${ }^{44}$ from homemade assembly mixtures (enzymes from New England Biolabs, NTPs and other chemicals from Sigma). O-methyltransferase protein sequence accession numbers and corresponding codon-optimized nucleotide sequences that were ordered as IDT gBlocks are also included in SI Table 3.

Additional description of methods (Chemicals, Culture conditions, Fluorescence measurements, and Metabolite analysis) can be found in Supporting Information. 


\section{Acknowledgements}

We acknowledge Professor George Church (Harvard Medical School, Boston, MA) for limited use of a fluorescent 96-well plate reader (Biotek H4 Synergy) and Spencer Wenck for assistance in constructing the original vanillate sensor. We are also grateful to Professor Chris Voigt (MIT, Cambridge, MA) for sensor evolution and for sharing evolved sensor constructs. This research was initially supported by the National Science Foundation through the Synthetic Biology Engineering Research Center (Synberc, Grant No. EEC0540879) and through a Graduate Research Fellowship to AMK. O-methyltransferase sequences were supported by a grant from the MIT-Portugal Program (Grant Number 6937814).

\section{Supporting Information}

Supporting Information is available free of charge.

Supporting Information (PDF) contains additional description of methods, a figure demonstrating sensor activation during pathway co-expression, and tables of plasmids, oligonucleotides, and synthetic gene (gBlock) sequences. 


\section{References}

(1) Lutz, R.; Bujard, H. Independent and Tight Regulation of Transcriptional Units in Escherichia Coli via the LacR/O, the TetR/O and AraC/I1-I2 Regulatory Elements. Nucleic Acids Res. 1997, 25 (6), 1203-1210.

(2) Brophy, J. A. N.; Voigt, C. A. Principles of Genetic Circuit Design. Nat Meth 2014, 11 (5), 508-520.

(3) Dietrich, J. A.; McKee, A. E.; Keasling, J. D. High-Throughput Metabolic Engineering: Advances in Small-Molecule Screening and Selection. Annu. Rev. Biochem. 2010, 79 (1), 563-590.

(4) Liu, D.; Evans, T.; Zhang, F. Applications and Advances of Metabolite Biosensors for Metabolic Engineering. Metab. Eng. 2015, 31, 35-43.

(5) Chou, H. H.; Keasling, J. D. Programming Adaptive Control to Evolve Increased Metabolite Production. Nat. Commun. 2013, 4, 2595.

(6) Schendzielorz, G.; Dippong, M.; Grünberger, A.; Kohlheyer, D.; Yoshida, A.; Binder, S.; Nishiyama, C.; Nishiyama, M.; Bott, M.; Eggeling, L. Taking Control over Control: Use of Product Sensing in Single Cells to Remove Flux Control at Key Enzymes in Biosynthesis Pathways. ACS Synth. Biol. 2014, 3 (1), 21-29.

(7) Raman, S.; Rogers, J. K.; Taylor, N. D.; Church, G. M. Evolution-Guided Optimization of Biosynthetic Pathways. Proc. Natl. Acad. Sci. 2014, 111 (50), 17803-17808.

(8) Zhang, F.; Carothers, J. M.; Keasling, J. D. Design of a Dynamic Sensor-Regulator System for Production of Chemicals and Fuels Derived from Fatty Acids. Nat Biotech 2012, 30 (4), 354-359.

(9) Xu, P.; Li, L.; Zhang, F.; Stephanopoulos, G.; Koffas, M. Improving Fatty Acids Production by Engineering Dynamic Pathway Regulation and Metabolic Control. Proc. Natl. Acad. Sci. U. S. A. 2014, 111 (31), 11299-11304.

(10) Doong, S. J.; Gupta, A.; Prather, K. L. J. Layered Dynamic Regulation for Improving Metabolic Pathway Productivity InEscherichia Coli. Proc. Natl. Acad. Sci. U. S. A. 2018, 115 (12), 2964-2969.

(11) Kunjapur, A. M.; Tarasova, Y.; Prather, K. L. J. Synthesis and Accumulation of Aromatic Aldehydes in an Engineered Strain of Escherichia Coli. J. Am. Chem. Soc. 2014, 136 (33), 11644-11654.

(12) Kunjapur, A. M.; Hyun, J. C.; Prather, K. L. J. Deregulation of S-Adenosylmethionine Biosynthesis and Regeneration Improves Methylation in the E. Coli de Novo Vanillin Biosynthesis Pathway. Microb. Cell Fact. 2016, 15 (1), 1.

(13) Biorefinery Products: Global Markets. BCC Research 2017.

(14) Walton, N. J.; Mayer, M. J.; Narbad, A. Vanillin. Phytochemistry 2003, 63 (5), 505-515.

(15) Fox, D. T.; Hotta, K.; Kim, C.-Y.; Koppisch, A. T. The Missing Link in Petrobactin Biosynthesis: AsbF Encodes a (-)-3-Dehydroshikimate Dehydratase. Biochemistry 2008, 47 (47), 12251-12253.

(16) Lautala, P.; Ulmanen, I.; Taskinen, J. Molecular Mechanisms Controlling the Rate and Specificity of Catechol O-Methylation by Human Soluble Catechol O-Methyltransferase. Mol. Pharmacol. 2001, 59 (2), 393-402.

(17) Hansen, E. H.; Møller, B. L.; Kock, G. R.; Bünner, C. M.; Kristensen, C.; Jensen, O. R.; Okkels, F. T.; Olsen, C. E.; Motawia, M. S.; Hansen, J. De Novo Biosynthesis of Vanillin in Fission Yeast 
(Schizosaccharomyces Pombe) and Baker's Yeast (Saccharomyces Cerevisiae). Appl. Environ. Microbiol. 2009, 75 (9), 2765-2774.

(18) Brochado, A. R.; Patil, K. R. Overexpression of O-Methyltransferase Leads to Improved Vanillin Production in Baker's Yeast Only When Complemented with Model-Guided Network Engineering. Biotechnol. Bioeng. 2013, 110 (2), 656-659.

(19) He, A.; Li, T.; Daniels, L.; Fotheringham, I.; Rosazza, J. P. N. Nocardia Sp. Carboxylic Acid Reductase: Cloning, Expression, and Characterization of a New Aldehyde Oxidoreductase Family. Appl. Environ. Microbiol. 2004, 70 (3), 1874-1881.

(20) Venkitasubramanian, P.; Daniels, L.; Rosazza, J. P. N. Reduction of Carboxylic Acids by Nocardia Aldehyde Oxidoreductase Requires a Phosphopantetheinylated Enzyme. J. Biol. Chem. 2007, 282 (1), 478-485.

(21) Jha, R. K.; Kern, T. L.; Fox, D. T.; M. Strauss, C. E. Engineering an Acinetobacter Regulon for Biosensing and High-Throughput Enzyme Screening in E. Coli via Flow Cytometry. Nucleic Acids Res. 2014, 42 (12), 8150-8160.

(22) de los Santos, E. L. C.; Meyerowitz, J. T.; Mayo, S. L.; Murray, R. M. Engineering Transcriptional Regulator Effector Specificity Using Computational Design and In Vitro Rapid Prototyping: Developing a Vanillin Sensor. ACS Synth. Biol. 2016, 5 (4), 287-295.

(23) Sana, B.; Chia, K. H. B.; Raghavan, S. S.; Ramalingam, B.; Nagarajan, N.; Seayad, J.; Ghadessy, F. J. Development of a Genetically Programed Vanillin-Sensing Bacterium for High-Throughput Screening of Lignin-Degrading Enzyme Libraries. Biotechnol. Biofuels 2017, 10 (1), 32.

(24) Morawski, B.; Segura, A.; Ornston, L. N. Repression of Acinetobacter Vanillate Demethylase Synthesis by VanR, a Member of the GntR Family of Transcriptional Regulators. FEMS Microbiol. Lett. 2000, 187 (1), 65-68.

(25) Thanbichler, M.; Iniesta, A. A.; Shapiro, L. A Comprehensive Set of Plasmids for Vanillate- and Xylose-Inducible Gene Expression in Caulobacter Crescentus. Nucleic Acids Res. 2007, 35 (20), e137-e137.

(26) Morabbi Heravi, K.; Lange, J.; Watzlawick, H.; Kalinowski, J.; Altenbuchner, J. Transcriptional Regulation of the Vanillate Utilization Genes (VanABK Operon) of Corynebacterium Glutamicum by VanR, a PadR-like Repressor. J. Bacteriol. 2015, 197 (5), 959-972.

(27) Iniesta, A. A.; García-Heras, F.; Abellón-Ruiz, J.; Gallego-García, A.; Elías-Arnanz, M. Two Systems for Conditional Gene Expression in Myxococcus Xanthus Inducible by Isopropyl- $\beta-D-$ Thiogalactopyranoside or Vanillate. J. Bacteriol. 2012, 194 (21), 5875-5885.

(28) Priefert, H.; Rabenhorst, J.; Steinbüchel, A. Molecular Characterization of Genes of Pseudomonas Sp. Strain HR199 Involved in Bioconversion of Vanillin to Protocatechuate. J. Bacteriol. 1997, 179 (8), 2595-2607.

(29) Kanaya, S.; Kudo, Y. Specificity of Promoter Consensus Sequences in Escherichia Coli Chromosome. Nucleic Acids Symp. Ser. 1991, No. 25, 41-42.

(30) Shimada, T.; Yamazaki, Y.; Tanaka, K.; Ishihama, A. The Whole Set of Constitutive Promoters Recognized by RNA Polymerase RpoD Holoenzyme of Escherichia Coli. PLoS One 2014, 9 (3). 
(31) Kunjapur, A. M.; Hyun, J. C.; Prather, K. L. J. Deregulation of S-Adenosylmethionine Biosynthesis and Regeneration Improves Methylation in the E. Coli de Novo Vanillin Biosynthesis Pathway. Microb. Cell Fact. 2016, 15 (1).

(32) Hansen, J.; Hansen, E. H.; Polur, H.; Sheridan, J. M.; Heal, J. R.; Hamilton, W. D. O. COMPOSITIONS AND METHODS FOR THE BIOSYNTHESIS OF VANILLAN OR VANILLIN BETA-D-GLUCOSIDE. US 20140245496 A1, 2014.

(33) Ellefson, J. W.; Meyer, A. J.; Hughes, R. A.; Cannon, J. R.; Brodbelt, J. S.; Ellington, A. D. Directed Evolution of Genetic Parts and Circuits by Compartmentalized Partnered Replication. Nat. Biotechnol. 2014, 32 (1), 97-101.

(34) Meyer, A. J.; Segall-Shapiro, T. H.; Voigt, C. A. Marionette: E. Coli Containing 12 Highly-Optimized Small Molecule Sensors. bioRxiv 2018, 285866.

(35) Kunjapur, A. M.; Cervantes, B.; Prather, K. L. J. Coupling Carboxylic Acid Reductase to Inorganic Pyrophosphatase Enhances Cell-Free in Vitro Aldehyde Biosynthesis. Biochem. Eng. J. 2016, 109.

(36) Kopf, M.; Klähn, S.; Pade, N.; Weingärtner, C.; Hagemann, M.; Voß, B.; Hess, W. R. Comparative Genome Analysis of the Closely Related Synechocystis Strains PCC 6714 and PCC 6803. DNA Res. 2014, 21 (3), 255-266.

(37) Kopf, M.; Klähn, S.; Voss, B.; Stüber, K.; Huettel, B.; Reinhardt, R.; Hess, W. R. Finished Genome Sequence of the Unicellular Cyanobacterium Synechocystis Sp. Strain PCC 6714. Genome Announc. 2014, 2 (4).

(38) Kopycki, J. G.; Stubbs, M. T.; Brandt, W.; Hagemann, M.; Porzel, A.; Schmidt, J.; Schliemann, W.; Zenk, M. H.; Vogt, T. Functional and Structural Characterization of a Cation-Dependent OMethyltransferase from the Cyanobacterium Synechocystis Sp. Strain PCC 6803. J. Biol. Chem. 2008, 283 (30), 20888-20896.

(39) Nelson, J. T.; Lee, J.; Sims, J. W.; Schmidt, E. W. Characterization of SafC, a Catechol 4-OMethyltransferase Involved in Saframycin Biosynthesis. Appl. Environ. Microbiol. 2007, 73 (11), 3575-3580.

(40) Siegrist, J.; Aschwanden, S.; Mordhorst, S.; Thöny-Meyer, L.; Richter, M.; Andexer, J. N. Regiocomplementary O-Methylation of Catechols by Using Three-Enzyme Cascades. ChemBioChem 2015, 16 (18), 2576-2579.

(41) Siegrist, J.; Netzer, J.; Mordhorst, S.; Karst, L.; Gerhardt, S.; Einsle, O.; Richter, M.; Andexer, J. N. Functional and Structural Characterisation of a Bacterial $O$-Methyltransferase and Factors Determining Regioselectivity. FEBS Lett. 2017, 591 (2), 312-321.

(42) Deschamps, P.; Zivanovic, Y.; Moreira, D.; Rodriguez-Valera, F.; López-García, P. Pangenome Evidence for Extensive Interdomain Horizontal Transfer Affecting Lineage Core and Shell Genes in Uncultured Planktonic Thaumarchaeota and Euryarchaeota. Genome Biol. Evol. 2014, 6 (7), 1549-1563.

(43) Sambrook, J.; Russell, D. W. Molecular Cloning: A Laboratory Manual; Cold Spring Harbor Laboratory Press: Cold Spring Harbor, N.Y., 2001.

(44) Gibson, D. G.; Young, L.; Chuang, R.-Y.; Venter, J. C.; Hutchison, C. A.; Smith, H. O. Enzymatic Assembly of DNA Molecules up to Several Hundred Kilobases. Nat. Methods 2009, 6 (5), 343- 
bioRxiv preprint doi: https://doi.org/10.1101/375287; this version posted July 23,2018 . The copyright holder for this preprint (which was not certified by peer review) is the author/funder. All rights reserved. No reuse allowed without permission.

345. 


\section{Figures}

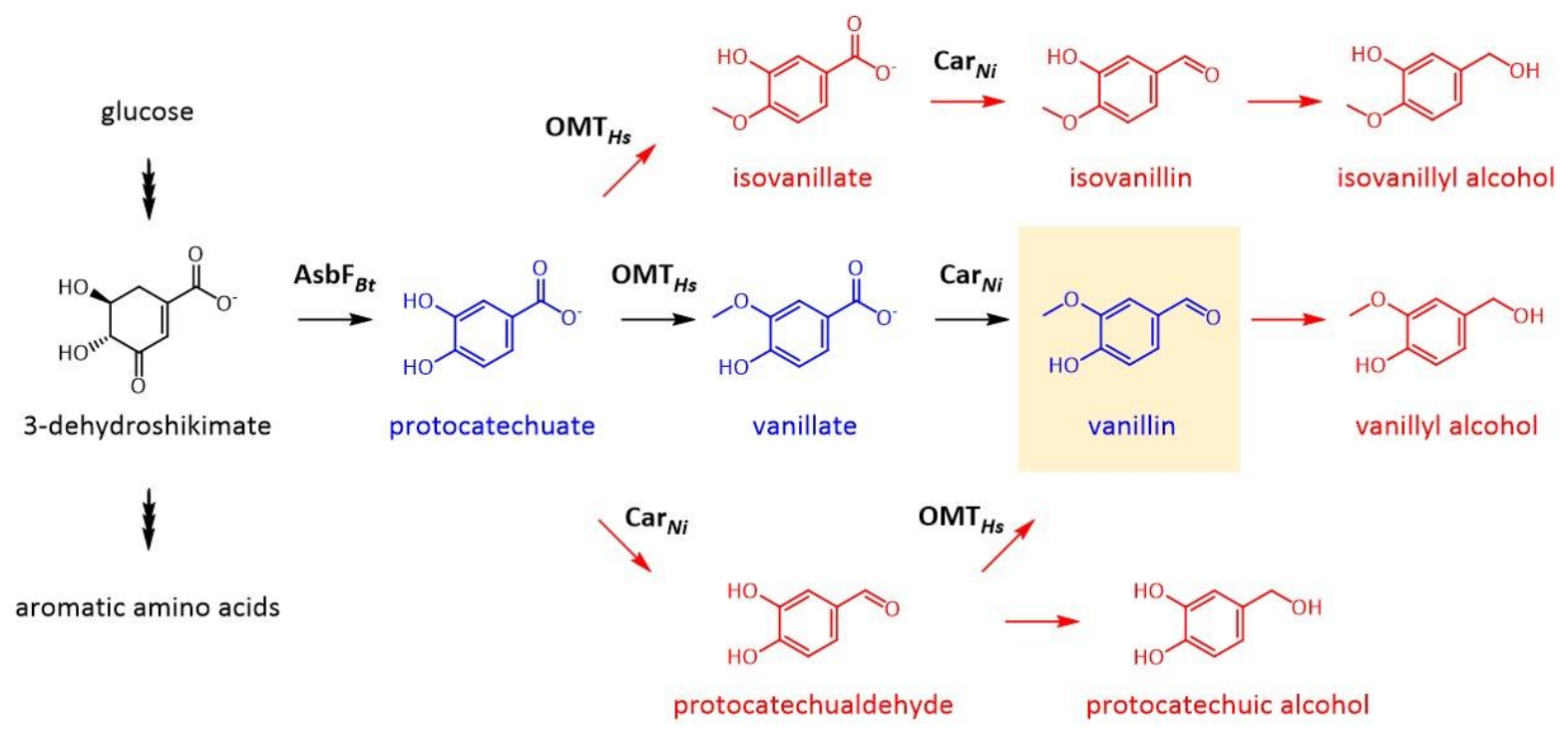

Figure 1. Metabolic pathway diagram of the engineered de novo vanillin biosynthesis pathway and potential byproducts in E. coli. Heterologous steps begin with AsbF-catalyzed conversion of endogenous 3-dehydroshikimate into protocatechuate. Protocatechuate, vanillate, and vanillin form desired heterologous metabolites and are shown in blue. Undesired metabolites that form due to low specificity of Car and OMT enzymes or due to endogenous aldehyde reductase activity in wild-type E. coli strains are shown in red. 

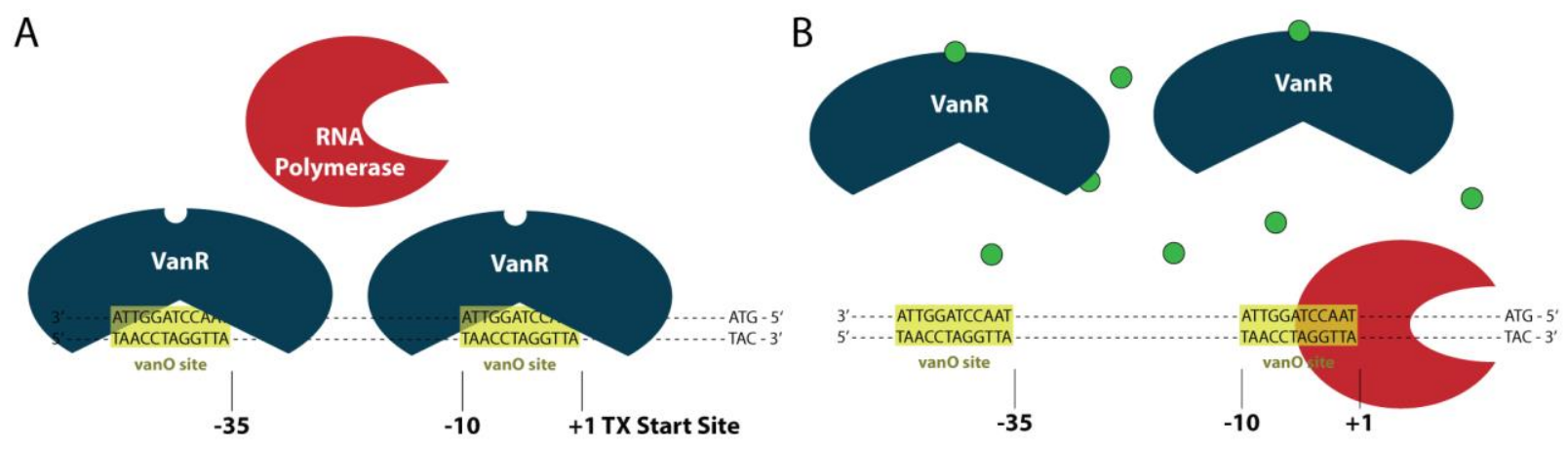

C

$-35$

$-10$

C. crescentus promoter: ATTGGATCCAATCTTGACGTCCGTTTGATTACGATCAAGATTGGATCCAAT

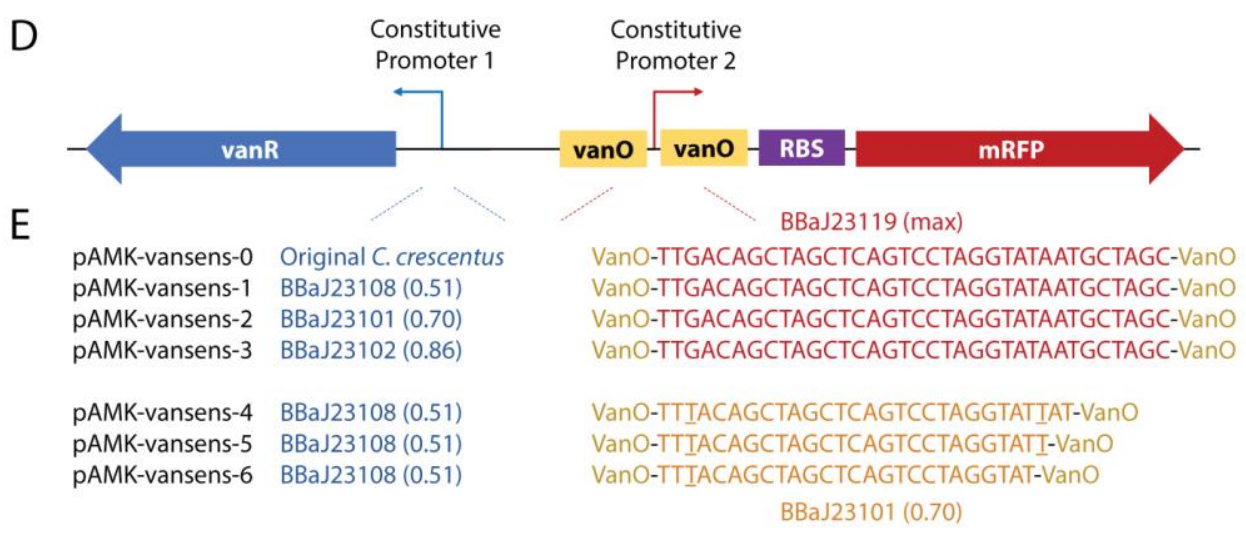

Figure 2. Original vanillate biosensor components and design. (A) Cartoon illustrating desired binding of vanillate repressor VanR to vanillate operator VanO sites. VanO sites are adjacent to -35 and -10 regions upstream of transcriptional start site, thereby hindering binding of sigma factor and RNA polymerase complex. (B) Cartoon illustrating desired unbinding of allosterically regulated VanR from VanO in the presence of vanillate, thereby enabling transcription. (C) Sequence of the native $p V a n A B$ promoter regulated by VanR in Caulobacter crescentus, showing that one of the two VanO sites overlaps with the -10 region. The consensus -10 region in E. coli is TATAAT and the consensus -35 is TTGACA. (D) High-level abstraction of vanillate sensor design. (E) Candidate promoter sequences tested experimentally in this manuscript. The two underlined " $T$ " $s$ in BBaJ23101 reflect the sequence difference between it and BBaJ23119. 
A

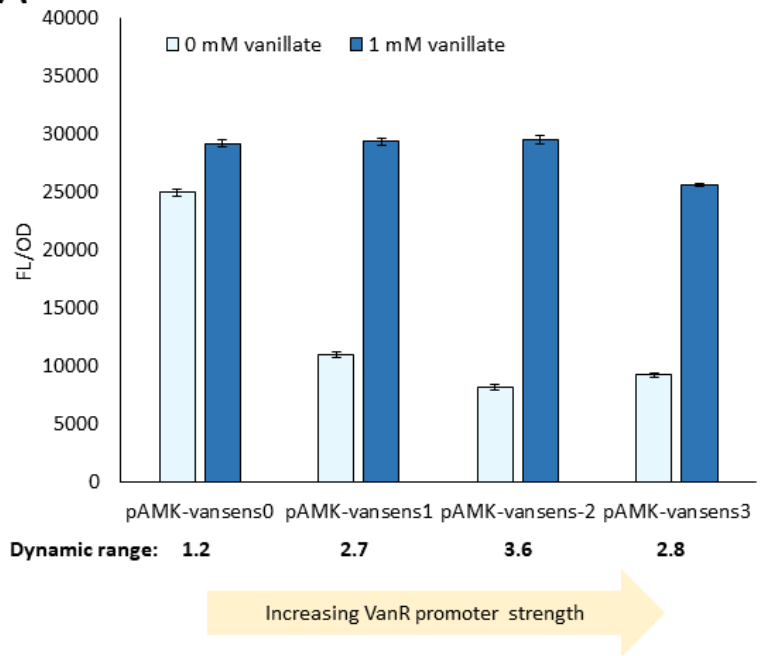

B

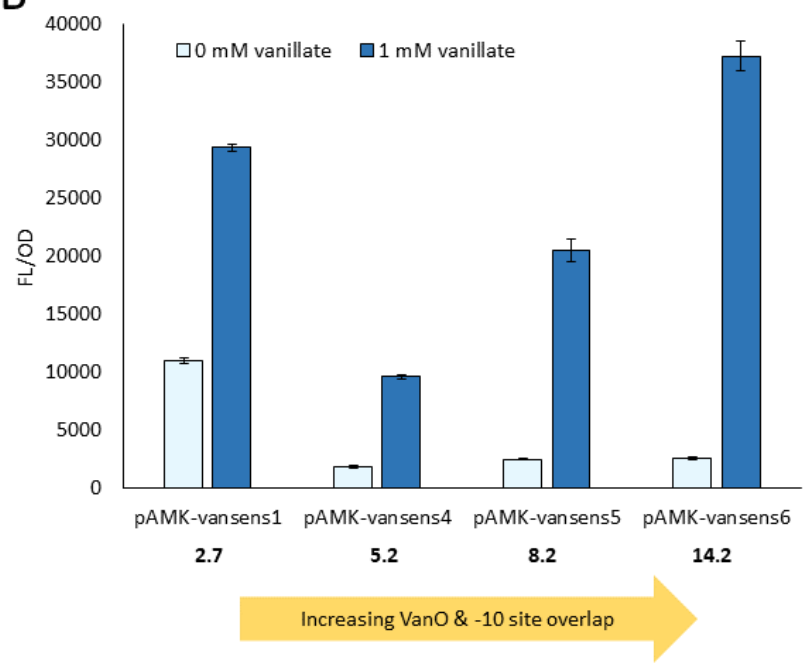

Figure 3. Dynamic range characterization of candidate vanillate sensor constructs. (A) Dynamic ranges observed using candidate vanillate sensor constructs expressing different VanR promoter strengths. (B) Dynamic ranges observed using constructs containing different extents of overlap between $V$ anO and $E$. coli -10 site. 

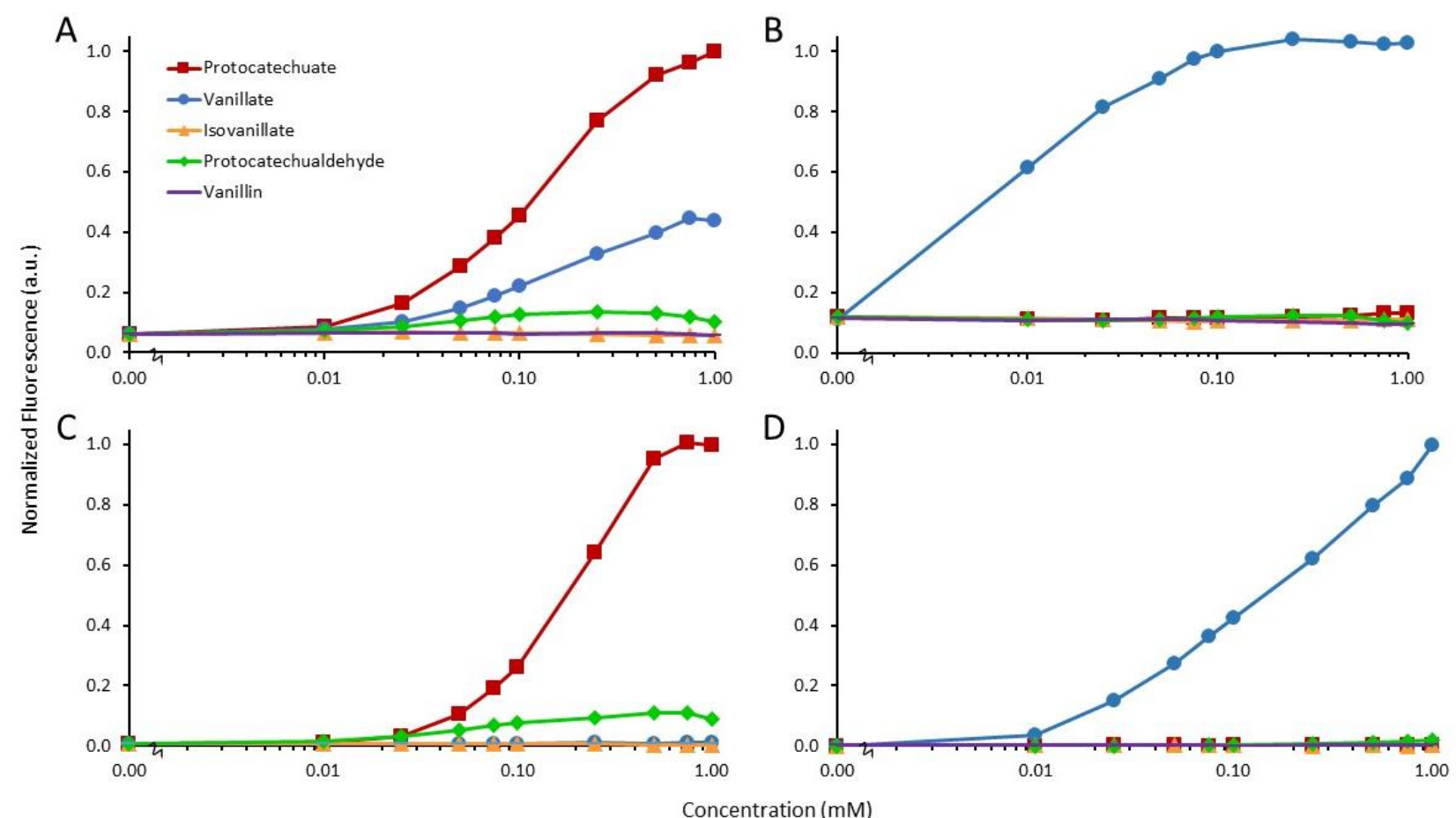

Figure 4. Dose-response characterization of original and evolved sensors intended for protocatechuate and vanillate, respectively. (A) Original protocatechuate sensor (from Ref. 21 and cloned into pAMK030). (B) Original vanillate sensor (pAMK022). (C) Evolved protocatechuate sensor (pAJM690, from Ref. 34). (D) Evolved vanillate sensor (pAJM773, from Ref. 34). 


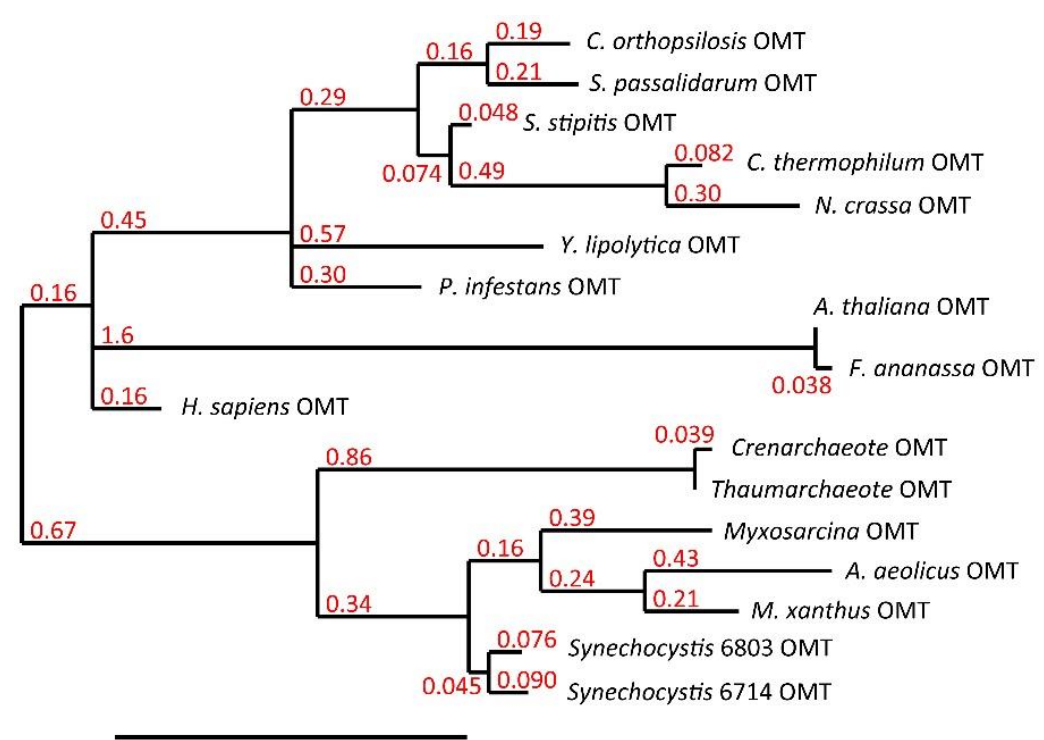

0.8

Figure 5. Phylogenetic tree of naturally occurring candidate OMT variants for biosensor-based bioprospecting. Sixteen variants from diverse sources across the tree of life were chosen for rapid screening of in vivo $O$-methyltransferase activity on exogenously supplemented protocatechuate. All are distant from the originally characterized $H$. sapiens OMT. Phylogenetic tree was generated using Phylogeny.fr and protein sequence inputs. Red values indicate branch lengths. 


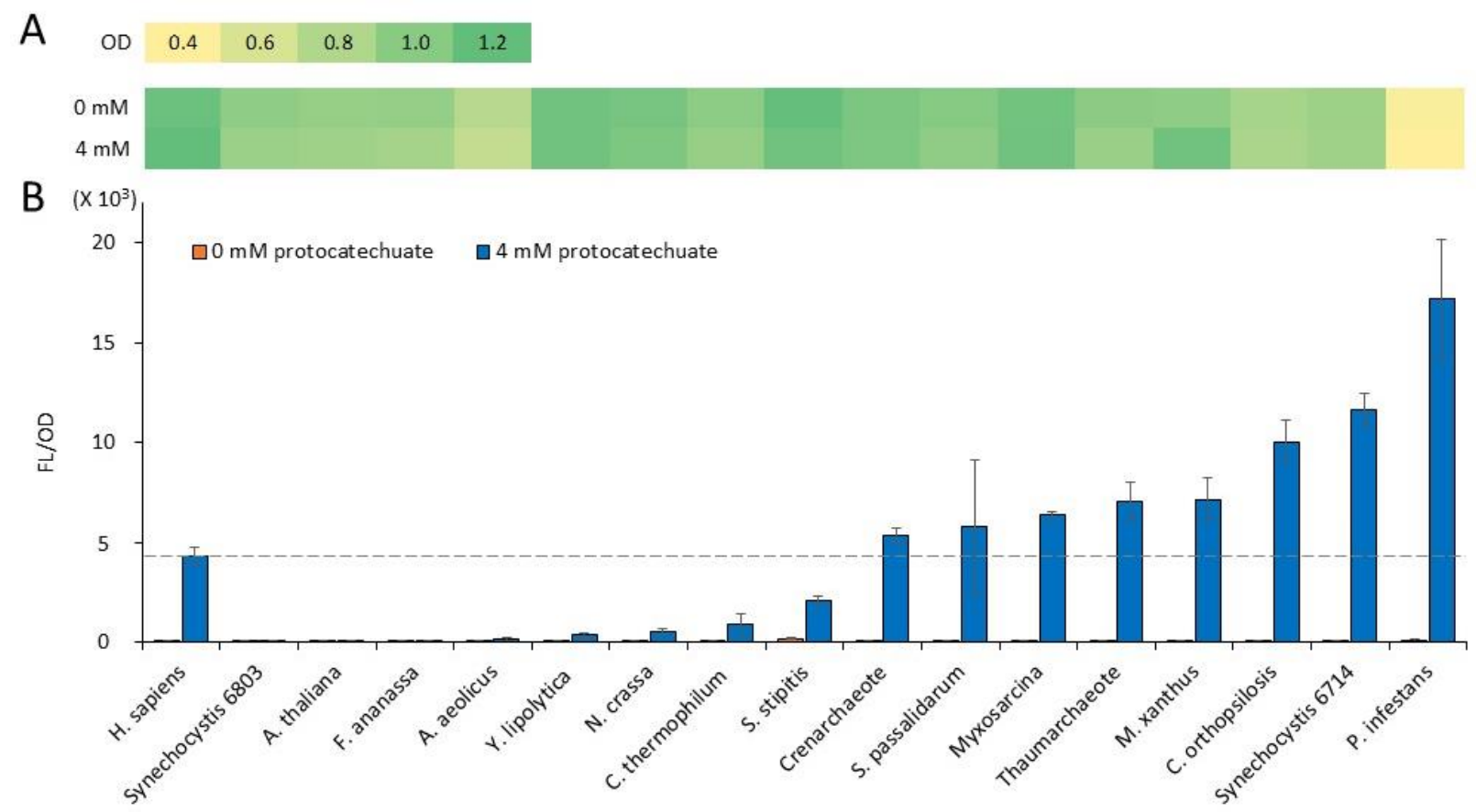

Figure 6. Biosensor-based bioprospecting of naturally occurring $\boldsymbol{O}$-methyltransferase variants. (A) Average final optical densities (OD) of cultures that harbor evolved vanillate biosensor and that express OMT variants in the absence $(0 \mathrm{mM})$ or presence $(4 \mathrm{mM})$ of exogenously supplied protocatechuate at the $24 \mathrm{~h}$ endpoint of the assay. Cultures that express $P$. infestans OMT achieved lower final ODs. (B) Endpoint OD-normalized fluorescence of cultures that harbor vanillate biosensor and that express OMT variants in the absence or presence of exogenously supplied protocatechuate. Dashed line demarcates performance of existing OMT to facilitate identification of improved variants. 